\title{
Heat stress attenuates skeletal muscle atrophy of extensor digitorum longus in streptozotocin-induced diabetic rats
}

\author{
K Nonaka ${ }^{1}$, S Une ${ }^{2}$, J Akiyama ${ }^{3}$ \\ ${ }^{1}$ Department of Physical Therapy, Osaka Prefecture University, Osaka, Japan \\ ${ }^{2}$ Department of Living Science, Shujitsu Junior College, Okayama, Japan \\ ${ }^{3}$ Department of Physical Therapy, Kibi International University, Takahashi, Japan
}

Received: May 1, 2015

Accepted after revision: June 14, 2015

\begin{abstract}
To investigate whether heat stress attenuates skeletal muscle atrophy of the extensor digitorum longus (EDL) muscle in streptozotocin-induced diabetic rats, 12-week-old male Wistar rats were randomly assigned to four groups ( $n=6$ per group): control (Con), heat stress (HS), diabetes mellitus (DM), and diabetes mellitus/heat stress (DM + HS). Diabetes was induced by intraperitoneal injection of streptozotocin $(50 \mathrm{mg} / \mathrm{kg})$. Heat stress was induced in the HS and DM + HS groups by immersion of the lower half of the body in hot water at $42{ }^{\circ} \mathrm{C}$ for 30 min; it was initiated 7 days after injection of streptozotocin, and was performed once a day, five times a week for 3 weeks. The muscle fiber cross-sectional area of EDL muscles from diabetic and non-diabetic rats was determined; heat stress protein (HSP) 72 and HSP25 expression levels were also analyzed by western blotting. Diabetes-induced muscle fiber atrophy was attenuated upon heat stress treatment in diabetic rats. HSP72 and HSP25 expression was upregulated in the DM + HS group compared with the DM group. Our findings suggest that heat stress attenuates atrophy of the EDL muscle by upregulating HSP72 and HSP25 expression.
\end{abstract}

Keywords: extensor digitorum longus muscle, heat stress, HSP72, HSP25, skeletal muscle atrophy, streptozotocin-induced diabetes

Diabetes mellitus (DM) induces skeletal muscle atrophy $(7,19)$ and decreases muscle strength $(7,9)$. Consequently, patients with diabetes have a higher risk of falls because of reduced muscle strength $(8,16)$ and DM-induced-muscle atrophy. Where DM induces skeletal muscle atrophy, fast-twitch fibers are prone to greater atrophy than are slow-twitch fibers (1). Fast-twitch fibers are responsible for high force/power/speed production and maintaining physical balance during postural perturbation. Hence, atrophy of fast-twitch muscle increases the risk of falling in DM patients. Therefore, an efficient strategy is required to attenuate muscle atrophy in order to reduce the risk of falls in DM patients.

Heat shock proteins (HSPs) have a protective effect on muscle atrophy. Senf et al. reported that HSP70 overexpression prevented rat soleus muscle fiber atrophy induced by hind limb immobilization (18). Furthermore, overexpression of HSP27 also prevented muscle fiber atrophy of the soleus muscle induced by disuse in rats (5). HSPs are expressed in response to several types of stress such as heat $(14,15)$ and exercise $(12)$. Exercise increased HSP72 expression (2) and attenuated skeletal muscle fiber atrophy (3) in streptozotocin

Corresponding author: Koji Nonaka

Department of Physical Therapy, Osaka Prefecture University

6-7-30 Habikino, Habikino, Osaka 583-8555, Japan

Phone: +81-72-950-2111; Fax: +81-72-950-2130; E-mail: nonaka@rehab.osakafu-u.ac.jp 
(STZ)-induced diabetic rats. These results suggest that upregulation of HSP72 protein induced by exercise may be responsible for attenuation of DM-induced muscle atrophy in diabetic rats.

The objective of the present study was to determine whether heat stress could attenuate skeletal muscle atrophy of the extensor digitorum longus (EDL) muscles, which are predominantly composed of fast-twitch fibers, in STZ-diabetic rats. We hypothesized that heat stress increases HSP72 and HSP25 expression and attenuates muscle atrophy in the EDL muscles of STZ-diabetic rats.

\section{Materials and Methods}

\section{Animals and heat stress}

All experimental procedures were performed in accordance with the Japanese Physiological Society Guide for the Care and Use of Laboratory Animals. The study was approved by the Institutional Animal Care and Use Committee of the Osaka Prefecture University. In total, 24 male Wistar rats (12 weeks old) were housed in a temperature-controlled room maintained at $22{ }^{\circ} \mathrm{C}$ under a 12-h light-dark cycle and were allowed free access to food and water. The rats were randomly divided into four groups with six animals per group: control (Con), heat stress (HS), STZ-induced diabetic (DM), and STZ-induced diabetic/heat stress (DM + HS). Diabetes was induced, according to methods described in a previous study (11), by a single intraperitoneal injection of STZ $(50 \mathrm{mg} / \mathrm{kg}$ body weight; Wako Pure Chemical Industries, Ltd., Osaka, Japan) dissolved in physiological saline. Equivalent amounts of the vehicle were injected into rats of the non-diabetic groups. After 7 days, blood was collected from the tail vein, and non-fasting blood glucose concentrations were measured using the Glutest Ace R (Sanwa Kagaku Kenkyusyo, Co., Ltd., Nagoya, Japan) and Glutest sensor (Sanwa Kagaku Kenkyusyo, Co., Ltd., Nagoya, Japan) kits. Rats with a blood glucose concentration $>300$ $\mathrm{mg} / \mathrm{dL}$ were defined as having diabetes.

Heat stress was performed according to a method reported in a previous study (13). The lower half of the body of the rat was immersed in hot water at $42{ }^{\circ} \mathrm{C}$ for $30 \mathrm{~min}$ under anesthesia (intraperitoneal injection of $50 \mathrm{mg} / \mathrm{kg}$ sodium pentobarbital). Harmful effects on the skin and signs of distress were not observed following heat stress. The heat stress procedure was initiated 7 days after the injection of STZ, and was performed once a day, five times a week for 3 weeks. To avoid any confounding effects of the anesthesia, the rats in the Con and DM groups were anesthetized similar to the rats in the HS and DM + HS groups.

Twenty-four hours after the final heat stress treatment, rats were euthanized by intraperitoneal injection of an overdose of sodium pentobarbital. Subsequently, the bilateral EDL muscles were removed, weighed immediately, frozen in isopentane cooled by liquid nitrogen, and stored at $-80{ }^{\circ} \mathrm{C}$ until further analysis.

\section{Analysis of the cross-sectional area of muscle fibers}

Muscles were sliced into $10-\mu \mathrm{m}$-thick cross-sections by using a cryostat at $-20{ }^{\circ} \mathrm{C}$. Sections were dried at room temperature for $30 \mathrm{~min}$, fixed in $10 \%$ formalin solution for $15 \mathrm{~min}$ at $4{ }^{\circ} \mathrm{C}$, and then stained with hematoxylin and eosin (H\&E). Stained sections were observed under a microscope, and images were obtained using a digital camera. The muscle fiber crosssectional areas of at least 800 fibers were measured by using Image J software (NIH, Bethesda, USA), and the mean of muscle fiber cross-sectional areas was determined. 


\section{Expression analysis of HSP 72 and HSP25}

The EDL muscles were homogenized in ice-cold Tris- $\mathrm{HCl}$ buffer $(50 \mathrm{mM}, \mathrm{pH} 7.4)$ containing cOmplete ${ }^{\mathrm{TM}}$ Protease Inhibitor Cocktail (Roche Diagnostics, Tokyo, Japan). Homogenates were centrifuged at $12,000 \mathrm{~g}$ for $10 \mathrm{~min}$ at $4{ }^{\circ} \mathrm{C}$, and aliquots of supernatants were used for subsequent analyses. The protein concentrations of the aliquots were determined using the Coomassie Protein Assay Kit (Thermo Fisher Scientific K.K., Yokohama, Japan) by the Bradford method. The absorbance was read at $595 \mathrm{~nm}$ by using a microplate reader (Model 680; Bio-Rad Laboratories, Inc., California, USA) to determine the protein concentration. EzApply (AE-1430; ATTO, Tokyo, Japan) was added to the aliquots, the final protein concentration was adjusted to $2 \mu \mathrm{g} / \mu \mathrm{l}$, and the samples were boiled at $95{ }^{\circ} \mathrm{C}$ for $5 \mathrm{~min}$. Subsequently, each protein sample was applied to a $12.5 \%$ polyacrylamide gel (E-R12.5L; ATTO, Tokyo, Japan), and electrophoresis was carried out at a constant current of $20 \mathrm{~mA} / \mathrm{gel}$ for $60 \mathrm{~min}$. The separated proteins were then transferred to a polyvinylidene difluoride membrane by using a HorizBLOT 2M (AE-6687; ATTO, Tokyo, Japan) system at a constant current of $2 \mathrm{~mA} / \mathrm{cm}^{2}$ for $60 \mathrm{~min}$. After transference, the polyvinylidene difluoride membranes were blocked with EzBlock Chemi (AE-1475; ATTO, Tokyo, Japan) for $1 \mathrm{~h}$ at room temperature, and were subsequently incubated overnight at $4{ }^{\circ} \mathrm{C}$ with the primary antibodies: anti-HSP72 (SPA-810; StressGen, Victoria, BC, Canada) diluted 1:20,000 in EzWash (AE1480; ATTO, Tokyo, Japan) with $0.1 \%$ Tween 20 (TTBS) and anti-HSP25 (SPA-801; StressGen, Victoria, BC, Canada) diluted 1:20,000 in TTBS. Following incubation, the membranes were washed three times for 10 min per wash in TTBS. They were then reacted for $1 \mathrm{~h}$ at room temperature with horseradish peroxidase-conjugated anti-mouse immunoglobulin G (IgG; 01803-44; Nacalai Tesque, Kyoto, Japan) diluted 1:25,000 in TTBS for HSP72 and anti-rabbit IgG (01827-44; Nacalai Tesque, Kyoto, Japan) diluted 1:25,000 in TTBS for HSP25. The membranes were further washed three times for 10 min per wash in TTBS, and were then reacted with ECL ${ }^{\text {TM }}$ Prime Western Blot Detection Reagent (RPN2232; CE Healthcare UK Ltd., Buckinghamshire, England) for $5 \mathrm{~min}$ at room temperature; protein bands were visualized with Hyperfilm ${ }^{\mathrm{TM}}$ ECL (28-9068-35; CE Healthcare UK Ltd., Buckinghamshire, England). Subsequently, the protein bands were scanned into a personal computer and analyzed using ImageJ software.

\section{Statistical analyse}

All data were expressed as the mean standard deviation (SD). The data were initially analyzed using one-way analysis of variance (ANOVA), and Tukey's post-hoc comparison test was used to assess significant differences between different groups. A $p$-value of $<0.05$ was considered significant.

\section{Results}

\section{Body weight and blood glucose}

The initial and final body weights and blood glucose levels are shown in Table I. The initial body weight was similar among all the groups, but the final body weight in the DM and DM + HS groups was significantly lower than that in the Con and HS groups $(p<0.0001)$. Furthermore, the final body weight in the HS group was significantly lower than that in the Con group $(p<0.001)$. 
Seven days after STZ injections, blood glucose levels were significantly higher in the $\mathrm{DM}$ and DM + HS groups than in the Con and HS groups $(p<0.0001)$. The blood glucose concentration of all the rats in the DM and DM + HS groups was $>300 \mathrm{mg} / \mathrm{dL}$.

Muscle weight

EDL muscle weight and EDL weight/body weight ratio are shown in Table I. EDL muscle weight in the DM and DM + HS groups was significantly decreased $(p<0.0001)$ compared with that in the Con group. The EDL muscle/body weight ratio was also significantly decreased in the DM group $(p<0.05)$, but not in the DM + HS group, relative to the ratio in the Con group.

Table I. Body weights, blood glucose levels and EDL muscle weights of the four groups

\begin{tabular}{|c|c|c|c|c|}
\hline & Con & HS & DM & DM + HS \\
\hline Initial body weight (g) & $286.6 \pm 5.4$ & $286.0 \pm 5.9$ & $285.3 \pm 13.4$ & $288.6 \pm 5.4$ \\
\hline Final body weight (g) & $333.3 \pm 8.2$ & $296.0 \pm 16.7^{* *}$ & $234.0 \pm 14.0^{* * *}, \# \#$ & $218.6 \pm 11.9^{* * *}, \# \#$ \\
\hline Blood glucose $(\mathrm{mg} / \mathrm{dl})$ & $141.3 \pm 65.6$ & $103.5 \pm 6.2$ & $435.3 \pm 45.4^{* * *}, \# \#$ & $515.0 \pm 66.9^{* * *}, \# \#$ \\
\hline EDL (mg) & $136.1 \pm 5.3$ & $129.0 \pm 6.4$ & $85.6 \pm 5.4^{* * *}, \# \#$ & $85.0 \pm 3.5^{* * *}, \# \#$ \\
\hline EDL weight/Body weight (mg/g) & $0.409 \pm 0.022$ & $0.436 \pm 0.020$ & $0.366 \pm 0.005^{*}, \# \#$ & $0.390 \pm 0.032^{\#}$ \\
\hline
\end{tabular}

Values are presented as means standard deviation (SD). $n=6$ per group. EDL, extensor digitorum longus; Con, control; HS, heat stress; DM, diabetes; DM + HS, diabetes and heat stress. $* p<0.05, * * p<0.001$,

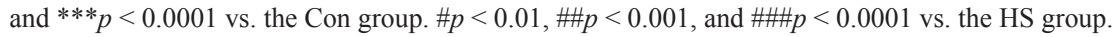

There were no significant differences $(p>0.05)$ between the DM and the DM + HS groups in any of these data

\section{Muscle fiber cross-sectional area}

The muscle fiber cross-sectional area of the EDL muscle was determined from H\&E-stained sections (Fig. 1). The cross-sectional area of the EDL muscle fiber was significantly decreased in the DM and DM + HS groups $(52 \%$ and $39 \%$, respectively; $p<0.001)$ relative to the area in the Con group. However, the EDL muscle fiber cross-sectional area in the DM group was significantly smaller than that in the DM + HS group $(p<0.05)$.

\section{HSP72 and HSP 25 expression}

The expression levels of HSP72 in the EDL muscles were determined by western blot analysis (Fig. 2a). There was no significant difference between the levels of HSP72 measured in the EDL muscles of the Con and DM groups. As expected, significant upregulation of HSP72 was observed in the EDL muscles of the HS group compared with those of the Con group $(p<0.0001)$. Furthermore, the expression levels of HSP72 in the EDL muscles of the DM + HS group were significantly higher than those in the EDL muscles of the DM group $(p<0.0001)$.

The expression levels of HSP25 in the EDL muscles were also determined by western blot analysis (Fig. 2b). No significant differences were observed between the HSP25 levels in EDL muscles from the Con and DM groups or the Con and HS groups. However, HSP25 expression was significantly upregulated in the EDL muscles of the DM + HS group compared with those in the DM group $(p<0.001)$. 


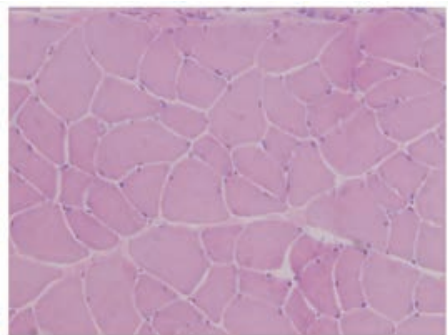

(a)

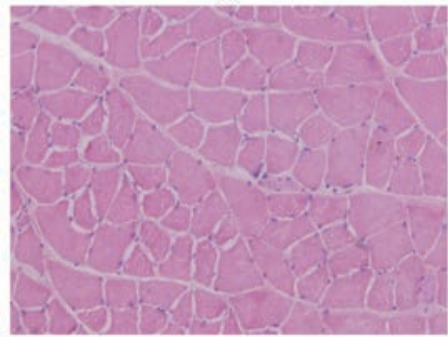

(c)

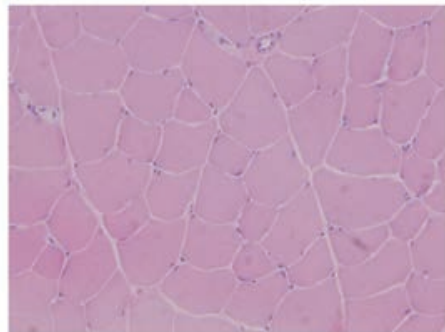

(b)

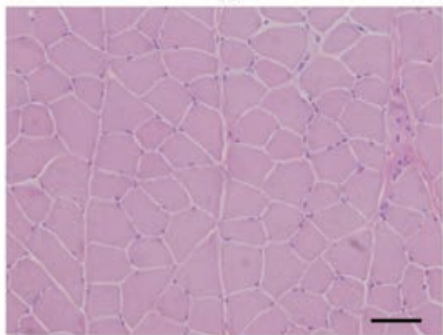

(d)

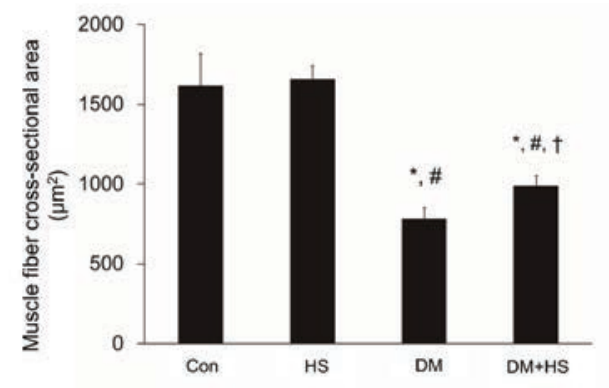

(e)

Fig. 1. Hematoxylin-eosin (H\&E) stained cross-sections and muscle fiber cross-sectional areas of the extensor digitorum longus (EDL) muscle. $n=6$ per group. Con, control; HS, heat stress; DM, diabetes; DM + HS, diabetes and heat stress. (a) Con group; (b) HS group; (c) DM group; (d) DM + HS group. (e) Graphical representation showing the mean muscle fiber cross-sectional area of the EDL muscle in the Con, HS, DM, and DM + HS groups. Values are expressed as mean \pm standard deviation (SD). ${ }^{*} p<0.0001$ vs. the Con group; $\# p<0.0001$ vs. the HS group; $\uparrow p<0.05$ vs. the DM group. Scale bar $=50 \mu \mathrm{m}$

\section{Discussion}

This study investigated the possibility that heat stress attenuates DM-induced skeletal muscle atrophy. We hypothesized that heat stress could prevent EDL muscle atrophy in STZ-induced diabetic rats. The major findings of the study were as follows: 1) DM-induced effects on EDL muscle weight/body weight ratio and muscle fiber atrophy were attenuated by heat stress, and 2) HSP72 and HSP25 protein levels were increased by heat stress. 


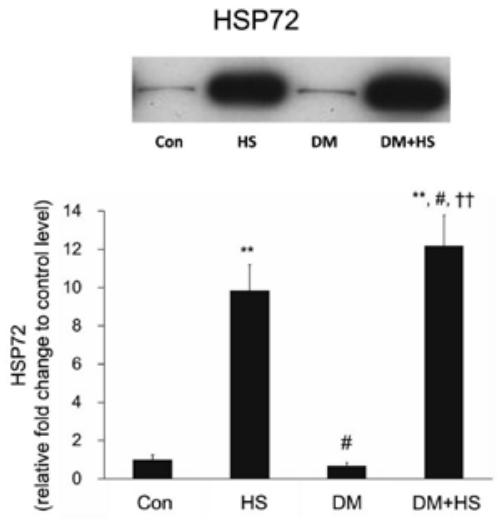

(a)

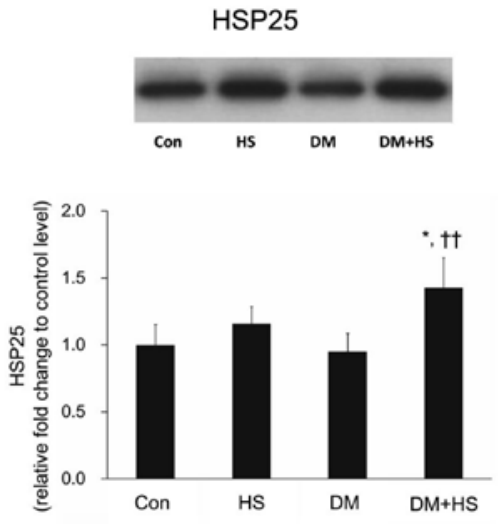

(b)

Fig. 2. Expression levels of heat shock protein (HSP) 72 (a) and HSP25 (b) in the EDL muscle. $n=6$ per group. Con, control; HS, heat stress; DM, diabetes; DM + HS, diabetes and heat stress. The expression pattern and protein levels of HSP72 in the EDL muscle of the Con, HS, DM, and DM + HS groups (a).

The expression pattern and protein levels of HSP25 in the EDL muscle of the Con, HS, DM, and DM + HS groups (b). Values are expressed as mean \pm standard deviation (SD). ${ }^{*} p<0.01$ and ${ }^{* *} p<0.0001$ vs. the Con group; $\# p<0.01$ and \#\#p<0.0001 vs. the HS group; $\uparrow p<0.001$ and $\dagger \dagger p<0.0001$ vs. the DM group

In the DM group but not the DM + HS group, the EDL weight/body weight ratio of rats was lower than that of rats in the Con group. In contrast, muscle weight was decreased in both the DM and DM + HS groups. Moreover, the body weight of rats in the DM and DM + HS groups decreased; these results are consistent with the findings of previous studies on STZinduced diabetic rats $(1,11)$. It is well known that muscle weight is influenced by body weight. Therefore, the weight of the EDL in the DM + HS group probably decreased with the reduction in body weight. In the DM group, the decrease in muscle weight appeared to be influenced not only by body weight but also by other factors such as increased loss of protein and water from skeletal muscle. These findings suggest that heat stress attenuates the decrease of EDL muscle mass in DM rats. Selsby et al. (17) reported that heat stress attenuates the reduced soleus weight/body weight ratio of rats observed during hind limb immobilization; our data are consistent with their results. Although the muscle fiber cross-sectional area of the EDL muscle decreased in both the DM and DM + HS groups relative to the cross-sectional area in the Con group, atrophy was higher in the DM group than in the DM + HS groups. These results suggest that heat stress may prevent muscle fiber degradation under diabetic conditions.

The expression levels of HSP72 and HSP25 were found to be elevated in the EDL muscle of the DM + HS group compared with levels in the Con and DM groups. In a previous study, heat stress applied to hind limb-immobilized rats increased HSP72 and HSP25 levels in the soleus muscle (17); our findings are consistent with these results. In addition, Najemnikova et al. reported that HSP72 levels were increased in the gastrocnemius and plantaris muscles, which are predominantly fast-twitch muscle fibers, of STZ-induced diabetic rats subjected to heat stress followed by 24-h recovery (10). Our results for HSP72 are consistent with these findings; however, we obtained contradictory results for HSP25. One possible reason for the discrepancy of HSP25 expression may be the different durations of heat stress in the two studies. Najemnikova et al. applied a single bout of heat stress in 
STZ-induced diabetic rats, whereas repeated bouts of heat stress were applied in the present study. Our results suggest that repeated heat-stress application in STZ-induced diabetic rats increases HSP25 expression in the skeletal muscle.

Heat stress induced a higher elevation of HSP72 levels in the DM + HS group than in the HS group. Moreover, heat stress failed to elevate HSP25 in the HS group, but it induced HSP25 elevation in the DM + HS group. These findings suggest that overall stress was greater in diabetic rats than in non-diabetic rats when heat stress was applied. Diabetes is known to increase oxidative stress in skeletal muscle (11); hence, diabetic rats may have suffered greater oxidative stress in addition to the applied heat stress, and consequently their overall stress was increased relative to that of non-diabetic rats. This may explain the higher levels of HSPs observed in the DM + HS group compared with the HS group.

Muscle atrophy is caused by an increased rate of proteolysis, and an increase in oxidative stress induces protein oxidation and accelerates proteolysis (4). In our previous study, we demonstrated that oxidative stress is increased in the EDL muscles of STZ-induced diabetic rats (11). Heat stress provides protection against oxidative stress and preserves muscle mass during hind limb immobilization (17). HSP25 overexpression protects $\mathrm{C} 2 \mathrm{C} 12$ myotubes against hydrogen peroxide-induced damage (6). Hence, our results in the present study suggest that high levels of HSP72 and HSP25 induced by heat stress reduce the rate of proteolysis caused by oxidative stress, which results in prevention of muscle atrophy under diabetic conditions. However, further studies are required to confirm this hypothesis.

Although our findings provide valuable information on the protective effect of heat stress on DM-induced muscle atrophy, this study has some limitations. First, we did not measure the rate of protein synthesis and protein degradation in skeletal muscle. Skeletal muscle atrophy is determined by the net balance between protein synthesis and protein degradation. Therefore, we could not elucidate the mechanisms underlying the preventive effect of heat stress on DM-induced muscle atrophy. Second, although we measured muscle weight and muscle fiber cross-sectional area, we did not measure skeletal muscle contractile force directly. Further studies are needed to elucidate the underlying mechanisms and the changes in skeletal muscle contractile force.

In conclusion, our results suggest that heat stress could be used as an adjuvant to rehabilitation for preventing muscle atrophy in diabetic patients. Future studies are necessary to determine whether heat stress has protective effects on skeletal muscle atrophy in patients with DM as it does in animal models.

\section{Acknowledgment}

This study was supported by the Project for Young Researcher Development of the Osaka Prefecture University.

\section{REFERENCES}

1. Armstrong RB, Gollnick PD, Ianuzzo CD: Histochemical properties of skeletal muscle fibers in streptozotocindiabetic rats. Cell Tissue Res. 162, 387-394 (1975)

2. Atalay M, Oksala NK, Laaksonen DE, Khanna S, Nakao C, Lappalainen J, Roy S, Hänninen O, Sen CK: Exercise training modulates heat shock protein response in diabetic rats. J. Appl. Physiol. 97, 605-611 (2004)

3. Chen GQ, Mou CY, Wang S, Zhao ZW: Exercise training has beneficial anti-atrophy effects by inhibiting oxidative stress-induced MuRF1 upregulation in rats with diabetes. Life Sci. 89, 44-49 (2011) 
4. Dean RT, Fu S, Stocker R, Davies MJ: Biochemistry and pathology of radical-mediated protein oxidation. Biochem. J. 324, 1-18 (1997)

5. Dodd SL, Hain B, Senf SM, Judge AR: Hsp27 inhibits IKKbeta-induced NF-kappaB activity and skeletal muscle atrophy. FASEB J. 23, 3415-3423 (2009)

6. Escobedo J, Pucci AM, Koh TJ: HSP25 protects skeletal muscle cells against oxidative stress. Free Radic. Biol. Med. 37, 1455-1462 (2004)

7. Leenders M, Verdijk LB, van der Hoeven L, Adam JJ, van Kranenburg J, Nilwik R, van Loon LJ: Patients with type 2 diabetes show a greater decline in muscle mass, muscle strength, and functional capacity with aging. J. Am. Med. Dir. Assoc. 14, 585-592 (2013)

8. Macgilchrist C, Paul L, Ellis BM, Howe TE, Kennon B, Godwin J: Lower-limb risk factors for falls in people with diabetes mellitus. Diabet. Med. 27, 162-168 (2010)

9. Maurer MS, Burcham J, Cheng H: Diabetes mellitus is associated with an increased risk of falls in elderly residents of a long-term care facility. J. Gerontol. A Biol. Sci. Med. Sci. 60, 1157-1162 (2005)

10. Najemnikova E, Rodgers CD, Locke M: Altered heat stress response following streptozotocin-induced diabetes. Cell Stress Chaperon. 12, 342-352 (2007)

11. Nonaka K, Une S, Tatsuta N, Ito K, Akiyama J: Changes in antioxidant enzymes and lipid peroxidation in extensor digitorum longus muscles of streptozotocin-diabetic rats may contribute to muscle atrophy. Acta Physiol. Hung. 101, 421-428 (2014)

12. Ogata T, Oishi Y, Higashida K, Higuchi M, Muraoka I: Prolonged exercise training induces long-term enhancement of HSP70 expression in rat plantaris muscle. Am. J. Physiol. Regul. Integr. Comp. Physiol. 296, R1557-R1563 (2009)

13. Oishi Y, Hayashida M, Tsukiashi S, Taniguchi K, Kami K, Roy RR, Ohira Y: Heat stress increases myonuclear number and fiber size via satellite cell activation in rat regenerating soleus fibers. J. Appl. Physiol. 107, 16121621 (2009)

14. Oishi Y, Taniguchi K, Matsumoto H, Ishihara A, Ohira Y, Roy RR: Differential responses of HSPs to heat stress in slow and fast regions of rat gastrocnemius muscle. Muscle Nerve 28, 587-594 (2003)

15. Oishi Y, Taniguchi K, Matsumoto H, Ishihara A, Ohira Y, Roy RR: Muscle type-specific response of HSP60, HSP72, and HSC73 during recovery after elevation of muscle temperature. J. Appl. Physiol. 92, 1097-1103 (2002)

16. Patel S, Hyer S, Tweed K, Kerry S, Allan K, Rodin A, Barron J: Risk factors for fractures and falls in older women with type 2 diabetes mellitus. Calcif. Tissue Int. 82, 87-91 (2008)

17. Selsby JT, Dodd SL: Heat stress reduces oxidative stress and protects muscle mass during immobilization. Am. J. Physiol. Regul. Integr. Comp. Physiol. 289, R134-R139 (2005)

18. Senf SM, Dodd SL, McClung JM, Judge AR: Hsp70 overexpression inhibits NF-kappaB and Foxo3a transcriptional activities and prevents skeletal muscle atrophy. FASEB J. 22, 3836-3845 (2008)

19. Severinsen K, Obel A, Jakobsen J, Andersen H: Atrophy of foot muscles in diabetic patients can be detected with ultrasonography. Diabetes Care 30, 3053-3057 (2007) 